\title{
ON A CONJECTURE OF ERDŐS, GALLAI, AND TUZA
}

\author{
GREGORY J. PULEO
}

\begin{abstract}
Erdős, Gallai, and Tuza posed the following problem: given an $n$-vertex graph $G$, let $\tau_{1}(G)$ denote the smallest size of a set of edges whose deletion makes $G$ triangle-free, and let $\alpha_{1}(G)$ denote the largest size of a set of edges containing at most one edge from each triangle of $G$. Is it always the case that $\alpha_{1}(G)+\tau_{1}(G) \leq n^{2} / 4$ ?

We have two main results. We first obtain the upper bound $\alpha_{1}(G)+\tau_{1}(G) \leq$ $5 n^{2} / 16$, as a partial result towards the Erdős-Gallai-Tuza conjecture. We also show that always $\alpha_{1}(G) \leq n^{2} / 2-m$, where $m$ is the number of edges in $G$; this bound is sharp in several notable cases.
\end{abstract}

\section{INTRODUCTION}

Given an $n$-vertex graph $G$, say that a set $A \subseteq E(G)$ is triangle-independent if it contains at most one edge from each triangle of $G$, and say that $X \subseteq E(G)$ is a triangle edge cover if $G \backslash X$ is triangle-free. Throughout this paper, $\alpha_{1}(G)$ denotes the maximum size of a triangle-independent set of edges in $G$, while $\tau_{1}(G)$ denotes the minimum size of a triangle edge cover in $G$.

Erdös [4] showed that every $n$-vertex graph $G$ has a bipartite subgraph with at least $|E(G)| / 2$ edges, which implies that $\tau_{1}(G) \leq|E(G)| / 2 \leq n^{2} / 4$. Similarly, if $A$ is triangle-independent, then the subgraph of $G$ with edge set $A$ is clearly triangle-free; by Mantel's Theorem, this implies that $\alpha_{1}(G) \leq n^{2} / 4$.

Intuitively, $\alpha_{1}(G)$ and $\tau_{1}(G)$ cannot both be large: if $\tau_{1}(G)$ is close to $n^{2} / 4$, then $|E(G)|$ is close to $n^{2} / 2$, which makes it difficult to find a large triangle-independent set of edges. Erdős, Gallai, and Tuza formalized this intuition with the following conjecture.

Conjecture 1.1 (Erdős-Gallai-Tuza [7]). For every n-vertex graph $G, \alpha_{1}(G)+$ $\tau_{1}(G) \leq n^{2} / 4$.

The conjecture is sharp, if true: consider the graphs $K_{n}$ and $K_{n / 2, n / 2}$, where $n$ is even. We have $\alpha_{1}\left(K_{n}\right)=n / 2$ and $\tau_{1}\left(K_{n}\right)=\left(\begin{array}{c}n \\ 2\end{array}\right)-n^{2} / 4$, while $\alpha_{1}\left(K_{n / 2, n / 2}\right)=n^{2} / 4$ and $\tau_{1}\left(K_{n / 2, n / 2}\right)=0$. In both cases, $\alpha_{1}(G)+\tau_{1}(G)=n^{2} / 4$, but a different term dominates in each case. As observed by Erdős, Gallai, and Tuza, the difficulty of the conjecture lies in the variety of graphs for which the conjecture is sharp: any proof of the conjecture would need to account for both $K_{n}$ and $K_{n / 2, n / 2}$ without any waste.

Erdős, Gallai, and Tuza [7] considered the conjecture on graphs for which every edge lies in a triangle, and proved that there is a positive constant $c$ such that $\alpha_{1}(G)+\tau_{1}(G) \leq|E(G)|-c|E(G)|^{1 / 3}$ and $\alpha_{1}(G)+\tau_{1}(G) \leq|E(G)|-c|V(G)|^{1 / 2}$ for such graphs. Aside from the original paper of Erdős, Gallai, and Tuza, no other work appears to have been done on the conjecture. The conjecture also appears as Problem 46 in [9, a list of unsolved combinatorial problems. 
In this paper, we present two partial results towards Conjecture 1.1.

In Section 2, we extend some ideas of Erdős, Faudree, Pach and Spencer 6] in order to obtain the bound $\alpha_{1}(G)+\tau_{1}(G) \leq 5 n^{2} / 16$. In Section 3 , we obtain the bound $\alpha_{1}(G) \leq n^{2} / 2-m$, where $m=|E(G)|$, and characterize the graphs for which equality holds. When $n$ is even, this bound is sharp for both $K_{n}$ and $K_{n / 2, n / 2}$, which makes it an encouraging step towards the Erdős-Gallai-Tuza Conjecture.

\section{Induced Bipartite Subgraphs}

In this section, we will focus on the relationship between triangle-free subgraphs of $G$ and bipartite subgraphs of $G$. The problem of finding a largest bipartite subgraph of a graph $G$ is well-studied, and clearly any bipartite subgraph of $G$ is triangle-free, so we can reasonably hope to apply some of the existing literature on bipartite subgraphs to our current problem (see [2] and [8] for surveys of this literature).

We define some useful notation. For any graph $G$, let $\tau_{\mathrm{B}}(G)$ denote the smallest size of an edge set $X$ such that $G \backslash X$ is bipartite, and let $b(G)$ denote the largest size of a vertex set $B$ such that $G[B]$ is bipartite. Clearly, $\tau_{1}(G) \leq \tau_{\mathrm{B}}(G)$, so we seek bounds on $\alpha_{1}(G)+\tau_{\mathrm{B}}(G)$. When $A \subseteq E(G)$, we will abuse notation slightly by identifying $A$ with the spanning subgraph of $G$ having edge set $A$. This yields notation like $N_{A}(v)$, referring to the neighborhood of a vertex $v$ in the spanning subgraph of $G$ with edge set $A$.

The relationship between $\tau_{1}(G)$ and $\tau_{\mathrm{B}}(G)$ has been studied before. Erdős 5 asked which graphs $G$ satisfy $\tau_{1}(G)=\tau_{\mathrm{B}}(G)$. The question was pursued by Bondy, Shen, Thomassé and Thomassen [3, who proved that $\tau_{1}(G)=\tau_{\mathrm{B}}(G)$ when $\delta(G) \geq 0.85|V(G)|$, and later by Balogh, Keevash, and Sudakov [1], who proved that $\tau_{1}(G)=\tau_{\mathrm{B}}(G)$ when $\delta(G) \geq 0.79|V(G)|$.

Erdős, Faudree, Pach, and Spencer [6] studied the problem of finding a largest bipartite subgraph of a triangle-free graph, using the observation that if $u v$ is an edge of a triangle-free graph, then $G[N(u) \cup N(v)]$ is an induced bipartite subgraph of $G$. Here, we use a similar observation:

Lemma 2.1. For any graph $G$, any triangle-independent set $A \subseteq E(G)$, and any edge $u v \in E(G)$,

$$
d_{A}(u)+d_{A}(v) \leq b(G) .
$$

Proof. Since $A$ is triangle-independent, the sets $N_{A}(u)$ and $N_{A}(v)$ are independent and disjoint. Hence $G\left[N_{A}(u) \cup N_{A}(v)\right]$ is bipartite with $d_{A}(u)+d_{A}(v)$ vertices.

Lemma 2.2. For any graph $G$,

$$
\alpha_{1}(G) \leq \frac{n b(G)}{4}
$$

Proof. Let $A$ be any triangle-independent subset of $E(G)$. Applying Lemma 2.1 to all edges in $A$ and summing together the resulting inequalities gives

$$
\sum_{u \in V(G)} d_{A}(u)^{2}=\sum_{u v \in A}\left[d_{A}(u)+d_{A}(v)\right] \leq|A| b(G) .
$$

By the Cauchy-Schwarz Inequality, we have

$$
\sum_{u v \in A} d_{A}(u)^{2} \geq \frac{4|A|^{2}}{n}
$$


The desired inequality follows.

Lemma 2.3. For any graph $G$,

$$
\tau_{\mathrm{B}}(G) \leq \frac{n^{2}}{4}-\frac{b(G)^{2}}{4}
$$

Proof. This is essentially the $\delta=0$ case of Proposition 2.5 of $[6$. We sketch a probabilistic proof here. Let $B$ be the vertex set of a largest bipartite induced subgraph of $G$. If we randomly place the vertices of $V(G) \backslash B$ into the partite sets of $B$ and delete all edges within the partite sets, the expected number of deleted edges is $\frac{1}{2}|E(G) \backslash E(G[B])|$; hence $G$ can be made bipartite by deleting at most this many edges. Thus,

$$
\tau_{\mathrm{B}}(G) \leq \frac{1}{2}|E(G) \backslash E(G[B])| \leq \frac{1}{2}\left[\left(\begin{array}{c}
n \\
2
\end{array}\right)-\left(\begin{array}{c}
b(G) \\
2
\end{array}\right)\right] \leq \frac{n^{2}}{4}-\frac{b(G)^{2}}{4,}
$$

where the second inequality uses the fact that for any vertex set $T \subseteq V(G)$, there are at most $\left(\begin{array}{c}n \\ 2\end{array}\right)-\left(\begin{array}{c}|T| \\ 2\end{array}\right)$ edges in $E(G) \backslash E(G[T])$.

Corollary 2.4. For any graph $G$,

$$
\alpha_{1}(G)+\tau_{1}(G) \leq \alpha_{1}(G)+\tau_{\mathrm{B}}(G) \leq \frac{5 n^{2}}{16} .
$$

Proof. From Lemma 2.2 and Corollary 2.3 we immediately have

$$
\alpha_{1}(G)+\tau_{\mathrm{B}}(G) \leq \frac{n^{2}}{4}+\frac{n b(G)}{4}-\frac{b(G)^{2}}{4 .}
$$

Since the product $x(n-x)$ is maximized when $x=n / 2$, this implies

$$
\alpha_{1}(G)+\tau_{\mathrm{B}}(G) \leq \frac{5 n^{2}}{16}
$$

as desired.

While the parameter $\tau_{\mathrm{B}}(G)$ has been extensively studied, the sum $\alpha_{1}(G)+\tau_{\mathrm{B}}(G)$ has not, to our knowledge, been previously studied. We have not found any graph $G$ with $\alpha_{1}(G)+\tau_{\mathrm{B}}(G)>|V(G)|^{2} / 4$, so we close with the following conjecture, which strengthens Conjecture 1.1

Conjecture 2.5. For every n-vertex graph $G, \alpha_{1}(G)+\tau_{\mathrm{B}}(G) \leq n^{2} / 4$.

Computer search suggests that Conjecture 2.5 is true for all graphs on at most 8 vertices.

\section{Bounding $\alpha_{1}(G)$}

In this section, we will obtain the bound $\alpha_{1}(G) \leq n^{2} / 2-m$, where $m=|E(G)|$. We first need one quick lemma.

Lemma 3.1. Let $G$ be an n-vertex graph, and let $A \subseteq E(G)$ be triangle-independent. For every edge $u v \in A$, we have $d_{A}(u) \leq n-d_{G}(v)$.

Proof. The set $A$ cannot contain any edge $u w$ where $w \in N_{G}(v)$, since then $A$ would contain two edges of the triangle $u v w$. Hence $N_{A}(u) \subseteq V(G) \backslash N_{G}(w)$.

The join of the graphs $G_{1}, \ldots, G_{t}$, written $G_{1} \vee \cdots \vee G_{t}$, is the graph obtained from the disjoint union $G_{1}+\cdots+G_{t}$ by adding all edges between vertices from different $G_{i}$. 
Theorem 3.2. For an n-vertex graph $G$ with $m$ edges,

$$
\alpha_{1}(G) \leq \frac{n^{2}}{2}-m
$$

Equality holds if and only if there exist $r_{1}, \ldots, r_{t} \geq 1$ such that $G \cong K_{r_{1}, r_{1}} \vee \cdots \vee$ $K_{r_{t}, r_{t}}$.

Proof. Let $A \subseteq E(G)$ be triangle-independent, and let $M$ be a maximal matching in $A$. We study the degree sum $\sum_{v \in V(G)} d_{A}(v)$ by splitting it into the sum $\sum_{v \in V(M)} d_{A}(v)+\sum_{v \notin V(M)} d_{A}(v)$.

For each $v$ covered by $M$, let $v^{\prime}$ be its mate in $M$. Applying Lemma 3.1 to both endpoints of each edge in $M$, we obtain the bound

$$
\sum_{v \in V(M)} d_{A}(v) \leq \sum_{v \in V(M)}\left[n-d_{G}\left(v^{\prime}\right)\right]=\sum_{v \in V(M)}\left[n-d_{G}(v)\right] .
$$

To bound $\sum_{v \notin V(M)} d_{A}(v)$, we first observe that the vertices not covered by $M$ form an independent set in $A$, since any edge joining such vertices could be added to obtain a larger matching.

Now let $v$ be any vertex not covered by $M$. For each edge $w w^{\prime} \in M$, if $v w \in A$ then $v w^{\prime} \notin E(G)$, since otherwise $A$ contains two edges of the triangle $v w w^{\prime}$. Hence $d_{A}(v) \leq n-1-d_{G}(v)$, since each $A$-edge $v w$ is witnessed by a non- $G$-edge $v w^{\prime}$ where $w^{\prime} \neq v$. Summing this inequality over all uncovered $v$ yields

$$
\sum_{v \notin V(M)} d_{A}(v) \leq \sum_{v \notin V(M)}\left[n-1-d_{G}(v)\right] \leq \sum_{v \notin V(M)}\left[n-d_{G}(v)\right] .
$$

Combining this with the bound on $\sum_{v \in V(M)} d_{A}(V)$ and applying the degree-sum formula for $G$ yields

$$
\sum_{v \in V(G)} d_{A}(v) \leq \sum_{v \in V(G)}\left[n-d_{G}(v)\right]=n^{2}-2 m .
$$

Applying the degree-sum formula for $A$ completes the proof of the first claim.

Now we characterize the graphs for which equality holds. First we argue that if $G \cong K_{r_{1}, r_{1}} \vee \cdots \vee K_{r_{t}, r_{t}}$, then $\alpha_{1}(G) \geq \frac{n^{2}}{2}-m$. Let $V_{i}$ be the subset of $V(G)$ containing the vertices of the $i$ th graph in this join, and let $A=E\left(G\left[V_{1}\right]\right) \cup \cdots \cup$ $E\left(G\left[V_{t}\right]\right)$. The set $A$ is triangle-independent: if $u v w$ is a triangle in $G$ with $u v \in A$, then $u v \in E\left(G\left[V_{i}\right]\right)$ for some $i$, so $u$ and $v$ have no common neighbors in $G\left[V_{i}\right]$, so that $u w \in A$ implies $v w \notin E(G)$ and vice versa. Thus,

$$
\alpha_{1}(G) \geq|A|=\sum_{i=1}^{t}\left(r_{i}\right)^{2}=\sum_{i=1}^{t}\left(r_{i}+2\left(\begin{array}{c}
r_{i} \\
2
\end{array}\right)\right)=\frac{n}{2}+2 \sum\left(\begin{array}{c}
r_{i} \\
2
\end{array}\right)=\frac{n^{2}}{2}-m .
$$

Next we show that these are the only graphs for which equality holds. Let $A$ be a triangle-independent subset of $G$ with $|A|=n^{2} / 2-m$. Since equality holds in (11), equality must also hold in (2) for every maximal matching $M \subseteq A$. This is only possible if the sum $\sum_{v \notin V(M)}\left[n-1-d_{G}(v)\right]$ is empty. Thus, all maximal matchings in $A$ are perfect matchings.

Let $P_{4}$ denote the path on four vertices. We claim that if $A$ contains an induced subgraph isomorphic to $P_{4}$, then $A$ contains a nonperfect maximal matching. Let $v_{1}, \ldots, v_{4}$ be the vertices of an induced copy of $P_{4}$ in $A$, written in order, and let $M$ be any maximal matching containing the edges $v_{1} v_{2}$ and $v_{3} v_{4}$; we may assume that 
$M$ is a perfect matching. Now $\left(M \backslash\left\{v_{1} v_{2}, v_{3} v_{4}\right\}\right) \cup\left\{v_{2} v_{3}\right\}$ is a nonperfect maximal matching, since $v_{1} v_{4} \notin A$.

Thus, if equality holds in (11), then $A$ is a triangle-free graph with a perfect matching and no induced $P_{4}$. This implies that every component of $A$ is a balanced complete bipartite graph.

Next we claim that if $u$ and $v$ are vertices in different components of $A$, then $u v \in E(G)$. Suppose $u v \notin E(G)$, and let $G^{\prime}=G+u v$. Now $A$ still contains at most one edge from any triangle of $G^{\prime}$ : if not, then $A$ contains two edges of $u v z$, for some $z \in V\left(G^{\prime}\right)$. Since $u v \notin A$, this implies that $u z \in A$ and $v z \in A$, contradicting the hypothesis that $u$ and $v$ are in different components of $A$. It follows that $|A| \leq n^{2} / 2-\left|E\left(G^{\prime}\right)\right|<n^{2} / 2-m$, contradicting the assumption that $|A|=n^{2} / 2-m$.

We have shown that the vertices of $G$ can be covered by vertex-disjoint complete bipartite graphs, and that if $u$ and $v$ are covered by different graphs, then $u v \in$ $E(G)$. This implies that $G \cong K_{r_{1}, r_{1}} \vee \cdots \vee K_{r_{t}, r_{t}}$.

\section{REFERENCES}

1. József Balogh, Peter Keevash, and Benny Sudakov, On the minimal degree implying equality of the largest triangle-free and bipartite subgraphs, J. Combin. Theory Ser. B 96 (2006), no. 6, 919-932. MR 2274084 (2007h:05080)

2. B. Bollobás and A. D. Scott, Better bounds for Max Cut, Contemporary combinatorics, Bolyai Soc. Math. Stud., vol. 10, János Bolyai Math. Soc., Budapest, 2002, pp. 185-246. MR 1919571 (2003g:05070)

3. Adrian Bondy, Jian Shen, Stéphan Thomassé, and Carsten Thomassen, Density conditions for triangles in multipartite graphs, Combinatorica 26 (2006), no. 2, 121-131. MR 2223630 (2007a:05062)

4. P. Erdős, On some extremal problems in graph theory, Israel J. Math. 3 (1965), 113-116. MR 0190027 (32 \#7443)

5. Paul Erdős, On some problems in graph theory, combinatorial analysis and combinatorial number theory, Graph theory and combinatorics (Cambridge, 1983), Academic Press, London, 1984, pp. 1-17. MR 777160 (86e:05001)

6. Paul Erdős, Ralph Faudree, János Pach, and Joel Spencer, How to make a graph bipartite, J. Combin. Theory Ser. B 45 (1988), no. 1, 86-98. MR 953897 (89f:05134)

7. Paul Erdős, Tibor Gallai, and Zsolt Tuza, Covering and independence in triangle structures, Discrete Math. 150 (1996), no. 1-3, 89-101, Selected papers in honour of Paul Erdős on the occasion of his 80th birthday (Keszthely, 1993). MR 1392722 (97d:05222)

8. Svatopluk Poljak and Zsolt Tuza, Maximum cuts and large bipartite subgraphs, Combinatorial optimization (New Brunswick, NJ, 1992-1993), DIMACS Ser. Discrete Math. Theoret. Comput. Sci., vol. 20, Amer. Math. Soc., Providence, RI, 1995, pp. 181-244. MR 1338615 (97a:90106)

9. Zsolt Tuza, Unsolved combinatorial problems part I, BRICS Lecture Series (2001), no. LS-01-1, $30-30$. 